\title{
Autologous Anti-mesothelin T-cell Receptor Fusion Construct T-cells TC-210
}

\author{
National Cancer Institute
}

\section{Source}

National Cancer Institute. Autologous Anti-mesothelin T-cell Receptor Fusion Construct

T-cells TC-210. NCI Thesaurus. Code C160780.

A preparation of autologous T-lymphocytes that have been genetically eng ineered to express a single-domain antibody that recognizes human mesothelin, fused to the $\mathrm{N}$ terminus of the CD3-epsilon T-cell receptor (TCR) subunit which, upon expression is incorporated into the endogenous TCR complex, with potential antineoplastic activity. Upon administration, the autologous anti-mesothelin TCR fusion construct (TRuC) T-cells TC-210 specifically target and bind to mesothelin-expressing tumor cells. This leads to T cell activation and T-cell mediated lysis of mesothelin-expressing tumor cells. Mesothelin, a cell surface glycoprotein involved in cell adhesion, is overexpressed in a variety of cancer cell types. Compared to chimeric antigen receptor (CAR) T-cells, TRuCs may be associated with less pro-inflammatory cytokine secretion and fewer adverse effects without compromising therapeutic efficacy. 